\title{
Community acquired pneumonia among adult patients at an Egyptian university hospital: bacterial etiology, susceptibility profile and evaluation of the response to initial empiric antibiotic therapy
}

This article was published in the following Dove Press journal: Infection and Drug Resistance

\author{
Rehab H El-Sokkary' \\ Raghdaa A Ramadan' \\ Mohamed El-Shabrawy ${ }^{2}$ \\ Lobna A El-Korashi' \\ Abeer Elhawary ${ }^{2}$ \\ Sameh Embarak ${ }^{2}$ \\ Rehab M Elsaid Tash' \\ Neveen G Elantouny ${ }^{3}$ \\ 'Medical Microbiology and \\ Immunology Department, Faculty of \\ Medicine, Zagazig University, Zagazig, \\ Egypt; ${ }^{2}$ Chest Department, Faculty of \\ Medicine, Zagazig University, Zagazig, \\ Egypt; ${ }^{3}$ Internal Medicine Department, \\ Faculty of Medicine, Zagazig \\ University, Zagazig, Egypt
}

Background: Effective empirical antibiotic therapy for community acquired pneumonia (CAP), based on frequently updated data about the pattern of bacterial distribution and their antimicrobial susceptibilities, is mandatory.

Aim: To identify the bacterial etiology of CAP in adults and their antibiotic susceptibility patterns and to evaluate the response to initial empirical antibiotic therapy in an Egyptian university hospital. Settings and design: A cross-sectional hospital-based study.

Patients and methods: CAP cases were selected by systemic random sampling from those admitted to the chest department. All were evaluated at admission and 4 days after starting empiric therapy. Typical bacteria were isolated, identified and tested for their antibiotic susceptibility. An indirect IF assay was used to diagnose atypical bacteria. Clinical response to initial empiric antibiotic therapy was clinically, laboratory and radiologically evaluated.

Results: Two hundred and seventy CAP patients were included. Bacteria represented $50.4 \%$ of them. Klebsiella pneumoniae was the most prevalent bacterium (10.37\%) followed by Streptococcus pneumoniae and P. aeruginosa (7.78\% each). Overall, $76.2 \%$ of isolates showed a multidrug resistant phenotype: 82.61\% (19/23) S. pneumoniae, $89.66 \%$ (26/29) K. pneumoniae, $65.22 \%$ (15/23) Pseudomonas aeruginosa, 87.50\% (7/8) Escherichia coli and $81.25 \%(13 / 16)$ Staphylococcus aureus. Broad spectrum $\beta$-lactams, especially carbapenems, and moxifloxacin showed in vitro efficacy on most of the tested isolates. Forty-three cases (15.9\%) were nonresponders, $37(86 \%)$ of them showed bacterial etiology. The highest rate of nonresponsiveness (30.43\%) was observed in cases receiving antipseudomonal/antipneumococcal $\beta$-lactam plus a fluoroquinolone for suspected $P$. aeruginosa infection.

Conclusion: Multidrug resistance in bacteria causing CAP and high frequency of isolation of hospital pathogens are prominent features of this study. Azithromycin containing regimens were associated with the lowest rates of nonresponsiveness. Development and implementation of an antibiotic stewardship program are highly recommended for CAP management.

Keywords: pneumonia, atypical bacteria, respiratory infection, community, antibiotic stewardship, empirical therapy, infection control

Correspondence: Rehab H El-Sokkary Medical Microbiology and Immunology Department, Faculty of Medicine, Zagazig University, 12 Abdaziz Ali Street, Zagazig Sharkeia, Egypt

Tel +201005650273

Fax +20 552307830

Email rehab_elsokkary@yahoo.com

\section{Introduction}

Community-acquired pneumonia (CAP) is an issue of public health concern, being a leading cause of morbidity that often requires hospitalization, and a significant cause 
of mortality, especially in severe cases presenting with sepsis or requiring assisted ventilation. ${ }^{1}$ Multiple agents can give rise to CAP but a few are responsible for the majority of cases, with bacterial pathogens accounting for a significant percentage of the cases. ${ }^{2}$ Despite the advances in management of CAP and the development of new diagnostic modalities, a definitive microbial etiology is not usually available for the first 3-4 days and clinical evaluation in the first two days is strongly recommended. ${ }^{3}$

In CAP, immediate initiation of effective antibiotic therapy is crucial for a favorable outcome, and empirical choice of initial antibiotic therapy is the mainstay of treatment. However, the rapid emergence and wide dissemination of microbial resistance have rendered most of the available antimicrobial agents ineffective. ${ }^{4}$

Evaluation of clinical response to initial treatment, ideally performed after 72 hours of starting treatment, is critical, as failure of such response carries a high risk of death. In this context, this study was conducted to identify the bacterial etiology of CAP in adults and their antibiotic susceptibility pattern and to evaluate the response to initial empiric antibiotic therapy in an Egyptian university hospital.

\section{Methods}

\section{Study design and setting}

This cross-sectional hospital-based study was conducted over a period of 27 months, from September 2015 to March 2018. It was carried out in Chest and Medical Microbiology and Immunology Departments, Zagazig University, Zagazig, Egypt.

\section{Patient selection and empirical antibiotic regimens}

This study included 270 patients diagnosed as having CAP, selected by systemic random sampling from those admitted to chest department. All were evaluated at admission and 4 days after starting empiric therapy.

CAP was defined as the presence of a new or progressive pulmonary infiltrate on chest radiograph, together with at least two of the following four criteria: fever $\left(>38.5^{\circ} \mathrm{C}\right)$, cough, production of purulent sputum or leukocytosis over $10,000 / \mathrm{mm}^{3}$. Those criteria had to be present before or within 48 hours of admission. Patients were excluded if aged less than 18 years, were discharged from a hospital within the 2 weeks preceding admission, were receiving antimicrobial therapy and/or immunosuppressive therapy, had computed tomography (CT) chest radiographic examination suggesting noninfectious causes such as pulmonary infarction, had acquired immunodeficiency syndrome or had leukemia. ${ }^{5}$

Initially, all patients received antibiotic therapy on their first day of admission on an empirical basis according to Infectious Diseases Society of America/American Thoracic Society (IDSA/ATS) 2007 guidelines: patients with severe CAP criteria were admitted to the intensive care unit (ICU), while others received ward care according to clinical evaluation in combination with patients' investigations, and severity scoring. ${ }^{6}$

The following antibiotic regimens were given for non-ICU patients: respiratory quinolones; levofloxacin or $\beta$-lactam (ampicillin-sulbactam [eg, IV q 6 hours]), cefotaxime ( $1 \mathrm{~g}$ IV q 8 hours) or ceftriaxone ( $1 \mathrm{~g}$ IV q 24 hours) combined with azithromycin (500 mg IV q 24 hours). For ICU admitted patients: intravenous combination of a potent antipneumococcal beta lactam (cefotaxime, ceftriaxone, or ampicillin sulbactam in full doses) plus an advanced macrolide (azithromycin) or plus respiratory fluoroquinolones (levofloxacin $750 \mathrm{mg}$ IV q 24 hours). For suspected Pseudomonas infection (presence of structural lung abnormalities eg, bronchiectasis, chronic obstructive pulmonary disease and a history of previous frequent antimicrobials or corticosteroids use) the following regimens were used: combined antibiotic therapy with an anti-pseudomonal/antipneumococcal beta lactam antibiotic ; cefepime ( $2 \mathrm{~g}$ IV q 12 hours), piperacillin-tazobactam (4.5 g IV q 6 hours), imipenem (500 mg IV q 6 hours), or meropenem ( 1 g IV q 8 hours) plus an antipseudomonal fluoroquinolones (levofloxacin $750 \mathrm{mg}$ IV q 24 hours) or an anti-pseudomonal/antipneumococcal beta lactam plus azithromycin (500 mg IV q 24 hours) and an aminoglycoside (gentamicin $7 \mathrm{mg} / \mathrm{kg} /$ day IV). Vancomycin (15 mg/kg IV q 12 hours) was added if methicillin resistant Staphylococcus aureus (MRSA) infection was suspected (either known MRSA colonization, or risk factors for it [eg, end stage renal disease, and injection drug users], presence of empyema, necrotizing or cavitary pneumonia, late flu like illness, or antimicrobial treatment, especially with fluoroquinolones, in the earlier three months). ${ }^{5,7}$

A minimum duration of treatment for non-ICU patients achieving an afebrile state for 48-72 hours was 5 days. Continuation of antibiotic therapy for longer duration was done if the initial treatment was not dynamic against the recognized pathogen or if the patient's condition was complicated by extra pulmonary infections. On the other hand, in patients admitted to ICU, the initial duration of antibiotic therapy was 7-10 days. 


\section{Patients' work-up}

Clinical evaluation

Cases were clinically assessed within 48 hours of hospital admission with full history taking and full clinical examination. Case severity was determined according to CURB-65 severity rating score for CAP: [C, mental confusion; $\mathrm{U}$, blood urea $>7 \mathrm{mmol} / \mathrm{L} ; \mathrm{R}$, respiratory rate $\geq 30 / \mathrm{min}$; $\mathrm{B}$, low blood pressure (diastolic $\leq 60 \mathrm{mmHg}$ or systolic $<90 \mathrm{mmHg}$ ); age $\geq 65$ years]. All included cases fulfilled a score of more than 1. Patients with a score of 0 or 1 are at low risk of death and were considered for home treatment. ${ }^{8}$

\section{Laboratory investigations}

Complete blood count (Sysmex ${ }^{\circledR}$ x 5 500; Kobe, Japan), kidney and liver function tests, serum electrolytes, C-reactive protein (COBAS INTEGRA ${ }^{\circledR} 400$; Hoffman-La Roche Ltd., Basel, Switzerland), arterial blood gas analysis (RAPIDLab ${ }^{\circledR}$ 348EX; Siemens, Munich, Germany).

\section{Radiological investigation}

All patients received a posteroanterior view plain chest X-ray (RotaLiX SRT 32; Philips, Italy) at admission and for follow up. When indicated, conventional $\mathrm{CT}$ was done by Hi-speed spiral CT (GE Medical Microsystem, Xi'an, China).

\section{Microbiological investigation}

Specimen collection

Before starting antibiotic treatment, blood and respiratory culture samples were collected. Blood culture samples were collected from all patients. Following careful alcohol skin disinfection, two samples of peripheral blood were drawn from two different venipuncture sites 30 minutes apart and were inoculated in blood culture bottles (Egyptian Diagnostic Media, Cairo, Egypt). Respiratory samples including sputum, endotracheal aspirates (ETA), bronchoalveolar-lavage (BAL) specimens, and pleural fluid (PF) were collected in some cases. In patients with productive cough, sputum samples were obtained at the time of initial clinical evaluation or within 24 hours of admission. If the patient was not able to expectorate sputum spontaneously, sputum was induced by $3 \%$ hypertonic saline nebulization. Regarding BAL specimens, around $20 \mathrm{~mL}$ of $0.9 \%$ saline solution were applied, during bronchoscopy under local anesthesia and collected through a fiber optic bronchoscope. An additional $5 \mathrm{~mL}$ of blood were drawn from each patient; sera were separated and stored at $-20^{\circ} \mathrm{C}$ for serological detection of atypical bacteria. Specimens were collected and transported under complete aseptic condition. ${ }^{8}$

\section{Identification of isolated bacteria}

For respiratory samples, both direct smear microscopy (Gram and Ziehl-Neelsen [ZN] stains) and bacterial culture were performed. High quality sputum and ETA (ie, 10 epithelial cells/low power field [LPF] and 25 white blood cells/LPF) and BAL specimens with significant growth of potential pathogens by quantitative cultures $\left(>10^{4}\right.$ colony forming units $/ \mathrm{mL}$ ) were included. Blood cultures were processed according to the standard methods. ${ }^{8}$ Positive acid-fast bacilli by ZN stain in the presence of suggestive radiological findings were diagnostic for pulmonary tuberculosis. Identification of isolated bacteria was done by conventional biochemical reactions. For Gram positive cocci, catalase, coagulase, optochin sensitivity tests were used. For Gram negative bacilli (GNB), the analytical profile index (API) (bioMérieux, Craponne, France); API $20 \mathrm{E}$ for enterobacteriacea and API 20NE for non-fermentative and oxidase tests were used. ${ }^{9}$ Atypical bacterial infections were diagnosed by indirect immunofluorescent technique for the detection of specific IgM antibodies to Mycoplasma pneumoniae, Chlamydophila pneumoniae, Legionella pneumophila serotype 1 and Coxiella burnetii using Pneumoslide-M test (Vircell, Granada, Spain). As per the manufacturer's instruction, phosphate buffered saline (PBS) was used to dilute the sera 1:1. Antihuman IgG sorbent was added to the diluted sera and incubated for 90 minutes at $37^{\circ} \mathrm{C}$ with the antigen-containing wells on the slide. After washing the slide with PBS, a fluorescent secondary IgM antibody was added to it and incubated at $37^{\circ} \mathrm{C}$ for 30 minutes, then washed again with PBS and finally read using fluorescence microscope at $400 \times$ magnification.

\section{Antibiotic susceptibility testing of isolated bacteria}

This was done by the modified Kirby-Bauer disk diffusion method on Muller Hinton agar (MHA) for selected antibiotics, including those commonly used for empirical therapy. ${ }^{10}$ Plates were incubated at $37^{\circ} \mathrm{C}$ for $16-18$ hours. MHA supplemented with 5\% sheep blood and Haemophilus test medium (HTM) were used for Streptococci and Haemophilus influenzae, respectively, and incubated in 5\% $\mathrm{CO} 2$. Cefoxitin was tested as a surrogate for oxacillin by disk diffusion method (cefoxitin disk $30 \mu \mathrm{g}$ ). As per CLSI recommendations, ${ }^{10} \mathrm{E}$-test (bioMérieux) was used to test for vancomycin susceptibility in Staphylococcus aureus and Streptococcus. This test measures the minimal inhibitory concentration (MIC) of the tested antibiotic. Screening for beta-lactam resistance in Streptococcus pneumoniae was done with the oxacillin $1 \mu \mathrm{g}$ disk; isolates with oxacillin 
$1 \mu \mathrm{g}$ disk zone diameter $\geq 20 \mathrm{~mm}$ are considered susceptible to benzylpenicillin, ampicillin, amoxicillin and piperacillin (without and with beta-lactamase inhibitor), cefotaxime, ceftriaxone and cefepime in addition to all carbapenems, while isolates with oxacillin $1 \mu \mathrm{g}$ disk zone diameter $<20 \mathrm{~mm}$ were further tested for MIC determination of these $\beta$-lactams. Oral penicillin breakpoints for $S$. pneumoniae were used. ${ }^{10}$

S. aureus ATCC 25923, Escherichia coli ATCC 25922 and Pseudomonas aeruginosa ATCC 27853 were used as quality control strains (American Type Culture Collection Global Bioresource Center, Manassas, VA, USA). Results of all susceptibility testing was interpreted according to CLSI guidelines. ${ }^{10}$

Non-susceptible isolates to at least one agent in three or more classes of antimicrobials were considered as multidrug resistant (MDR) isolates. MRSA is considered an MDR. ${ }^{11}$ MDR $S$. pneumoniae was defined as $S$. pneumoniae isolates showing nonsusceptibility to penicillin (MIC, $\geq 0.12 \mu \mathrm{g} / \mathrm{mL}$ ) and other $\geq 2$ non- $\beta$-lactam antimicrobial classes. ${ }^{12}$

\section{Clinical response to treatment}

Clinical response to treatment was evaluated within 48-72 hours of hospital admission. It was monitored by febrile chart, hemodynamics and chest radiography. Characteristics of patients showing an early response to treatment (defined as a time to clinical stability $\leq 4$ days) were compared with those of patients with failure of response to initial therapy. ${ }^{13}$

\section{Statistical analysis}

The data were coded, checked, entered and analyzed using SPSS version 15 for Windows (SPSS Inc., Chicago, IL, USA). Continuous variables are expressed as mean and SD. Categorical variables are expressed as frequencies and percent. Chi-square and Fisher's exact tests were used to examine the relationship between categorical variables. A significance level of $P<0.05$ was used in all tests.

The study was approved by the institutional review board (IRB) no 4759/5-8-2018, Faculty of medicine, Zagazig University. An informed written consent was obtained from all participants at time of recruitment. This study was conducted in accordance with the Declaration of Helsinki.

\section{Results}

\section{Patient characteristics}

Demographic, clinical, laboratory data and comorbidities of participants at admission are listed in Table 1.
Table I Characteristics of study participants $(n=270)$

\begin{tabular}{|c|c|c|}
\hline Characteristics & $\mathbf{n}$ & $\%$ \\
\hline Demographic data & & \\
\hline Age in years (mean $\pm S D$ ) & $56.7 \pm 16.3$ & \\
\hline Sex & & \\
\hline Female & 90 & 33.33 \\
\hline Male & 180 & 66.67 \\
\hline Habit & & \\
\hline Nonsmoker & 128 & 47.41 \\
\hline Cigarette smoking & 80 & 29.63 \\
\hline Goza smoking & 62 & 22.96 \\
\hline Drug and/or alcohol & 32 & 11.85 \\
\hline Clinical data & & \\
\hline Fever & 222 & 82.22 \\
\hline Dyspnea & 182 & 67.41 \\
\hline Cough & 214 & 79.26 \\
\hline Expectoration & 190 & 70.37 \\
\hline Hemoptysis & 80 & 29.63 \\
\hline Chest pain & 115 & 42.59 \\
\hline Laboratory data & & \\
\hline CRP, mg/dL & $176.43 \pm 3.46$ & \\
\hline WBC count, $\times 10^{9} / \mathrm{L}$ & $15.0 \pm 12.1$ & \\
\hline Platelet count, $\times 10^{9} / \mathrm{L}$ & $234 \pm 24.5$ & \\
\hline CURB-65 severity rate score & & \\
\hline CURB 2 & 185 & 68.52 \\
\hline CURB 3-5 & 85 & 31.48 \\
\hline Comorbidities & $(N=108)$ & 40.00 \\
\hline Diabetes mellitus & 34 & 31.48 \\
\hline Hypertension & 28 & 25.93 \\
\hline Ischemic heart disease & 18 & 16.67 \\
\hline Liver diseases & 8 & 7.40 \\
\hline COPD & 20 & 18.52 \\
\hline
\end{tabular}

Notes: Values are mean \pm SD or $n(\%)$.

Abbreviations: CRP, $C$ reactive protein; CURB-65 severity score [C, mental confusion; $U$, blood urea $>7 \mathrm{mmol} / \mathrm{L}$; $R$, respiratory rate $\geq 30 / \mathrm{min}$; $\mathrm{B}$, low blood pressure (diastolic $\leq 60 \mathrm{mmHg}$ or systolic $<90 \mathrm{mmHg}$ ); age $\geq 65$ years]; WBC, white blood cells.

\section{Bacterial etiology}

Out of 270 enrolled cases, 136 cases (50.4\%) showed bacterial etiology. Dual bacterial pathogens were identified in 9 cases $(3.33 \%)$, Table 2 .

\section{Antibiotic susceptibility testing}

Table 3 shows the antibiotic susceptibility pattern of 104 isolated bacterial agents. The single Streptococcus pyogenes isolate was susceptible to penicillin, and thus it was considered susceptible to amoxicillin-clavulanate, ampicillin-sulbactam, cefepime, cefotaxime, ceftriaxone, cefuroxime, imipenem and meropenem. The MDR phenotype was revealed in $76.19 \%(80 / 105)$ of tested bacteria, distributed as follows: $82.61 \%$ (19/23) S. pneumoniae, 89.66\% (26/29) Klebsiella pneumoniae, 65.22\% (15/23) P. aeruginosa, 87.50\% (7/8) E. coli and $81.25 \%(13 / 16) S$. aureus isolates were MRSA. 
Table 2 Bacterial etiology of CAP

\begin{tabular}{|c|c|c|c|c|c|}
\hline Bacterial etiology & $\mathbf{n}$ & $\%$ & $\begin{array}{l}\text { Blood } \\
\text { culture }\end{array}$ & $\begin{array}{l}\text { Respiratory } \\
\text { specimens }\end{array}$ & Serum $^{a}$ \\
\hline Single bacterial agent & 127 & 47.04 & & & \\
\hline K. pneumoniae & 28 & 10.37 & 12 & 16 & - \\
\hline S. pneumoniae & 21 & 7.78 & 12 & 9 & - \\
\hline P. aeruginosa & 21 & 7.78 & 0 & 21 & - \\
\hline M. pneumoniae & 15 & 5.56 & - & - & 15 \\
\hline S. aureus & 11 & 4.07 & 4 & 7 & - \\
\hline L. pneumophila & 10 & 3.70 & - & - & 10 \\
\hline E. coli & 8 & 2.96 & I & 7 & - \\
\hline H. influenzae & 5 & 1.85 & 0 & 5 & - \\
\hline C. pneumoniae & 4 & 1.48 & - & - & 4 \\
\hline M. tuberculosis & 4 & 1.48 & - & 4 & - \\
\hline Mixed bacterial agents & 9 & 3.33 & & & \\
\hline M. pneumoniae + S. aureus & 4 & 1.48 & 2 & 2 & $4^{\mathrm{b}}$ \\
\hline M. pneumoniae $+S$. pneumoniae & 1 & 0.37 & 1 & - & $\mathrm{I}^{\mathrm{b}}$ \\
\hline K. pneumoniae $+S$. aureus & I & 0.37 & I & I & \\
\hline L. pneumophilia $+P$. aeruginosa & 1 & 0.37 & - & $\mathrm{I}$ & $\mathrm{I}^{\mathrm{b}}$ \\
\hline L. pneumophilia + S. pyogenes & I & 0.37 & - & 1 & $\mathrm{I}^{\mathrm{b}}$ \\
\hline S. pneumoniae $+P$. aeruginosa & I & 0.37 & I & $\mathrm{I}$ & \\
\hline
\end{tabular}

Notes: aSerology testing was done for detection of atypical bacteria from serum. 'ंAtypical bacteria; M. pneumonia, L. pneumophilia.

Abbreviation: CAP, community acquired pneumonia; K. pneumoniae, Klebsiella pneumoniae; S. Pneumoniae; Streptococcus pneumoniae; $P$. aeruginosa, Pseudomonas aeruginosa; M. pneumoniae, Mycoplasma pneumoniae; S. aureus, Staphylococcus aureus; L. pneumophila, Legionella pneumophila; E. coli, Escherichia coli; H. influenzae, Haemophilus influenzae; C. pneumoniae, Chlamydophila pneumoniae; M. tuberculosis, Mycobacterium tuberculosis; S. pyogenes, Streptococcus pyogenes.

\section{Nonresponders}

Forty-three cases were diagnosed as nonresponders at a rate of (15.9\%); their characteristics are presented in Table 4. Mortality rate among responders was $11 / 227$ (4.85\%), and $7 / 43$ (16.3\%) among nonresponders.

\section{Discussion}

This study included 270 adult patients diagnosed with CAP. Smokers represented (52.5\%) of cases. Smoking is a reported risk factor for CAP. It increases the susceptibility to respiratory infection through disturbance of the host defense mechanisms. The association between smoking habits and CAP development was confirmed in previous studies. ${ }^{14} \mathrm{Co}$-morbid conditions were present in $40 \%$ of patients. Diabetes mellitus was the most common comorbidity followed by hypertension and ischemic heart diseases. Similar co-morbidities were previously reported..$^{15}$ This highlights chronic debilitating conditions, particularly diabetes, as risk factors in CAP.

Bacterial etiology was identified in $50.4 \%$ of enrolled cases. K. pneumoniae showed the highest rate of isolation $(10.37 \%)$ followed by $S$. pneumoniae and P. aeruginosa (7.78\% each) and atypical bacteria were the etiology of 36 cases $(13.3 \%)$. These rates differ from those previously reported by another Egyptian study on CAP in 2013 which reported $S$. pneumoniae as the most common bacterium (36.4\%), followed by S. aureus (7\%); K. pneumoniae, P. aeruginosa and E. coli at rates of 4.8, 2.1 and 1.6\%, respectively. ${ }^{16}$ Another Egyptian study in Upper Egypt reported
S. pneumoniae followed by atypical bacteria (C. pneumoniae and $M$. pneumoniae), then $K$. pneumoniae as the causative bacteria of adult CAP at rates of $36 \%, 30 \%$ and $10 \%$, respectively. ${ }^{17}$ Low rates of $S$. pneumoniae in our isolates may be a true reduction due to increased awareness of pneumococcal vaccine by susceptible population or due to lower sensitivity of the used conventional methods.

CAP has long been known to be caused by pathogens such as S. pneumoniae, H. influenzae and atypical pathogens that are sensitive to the majority of the first-line antibiotics. Recently, GNB that used to dominate the hospital environment, such as P. aeruginosa, K. pneumoniae and E. coli, have emerged as causes of CAP with an estimated prevalence ranging from $2 \%$ to $30 \% .{ }^{18}$ Our findings are in line with this perspective, where GNB account for around $21 \%$ of CAP cases. The appreciation of GNB role in CAP that has elevated over the past few decades is probably due to an increase in the number of old CAP patients who are usually harboring colonizers of GNB, in addition to the reported high severity of illness caused by GNB that usually require hospital, and mostly ICU admission. ${ }^{19}$

$K$. pneumoniae, the commonly isolated bacterium in this study, showed high resistance to penicillin/ $\beta$-lactamase inhibitors, except for piperacillin/tazobactam. Resistance rates to third and fourth generation cephalosporins ranged from $65.5 \%$ to $37.9 \%$. Conversely, carbapenems showed the highest susceptibility among the tested $\beta$-lactams. Of K. pneumoniae isolates $41.4 \%$ and $34.5 \%$ were resistant 
Table 3 Frequency of antibiotic resistance in bacterial isolates $(n=104)$

\begin{tabular}{|c|c|c|c|c|c|c|}
\hline & $\begin{array}{l}\text { S. pneumoniae } \\
(n=23)\end{array}$ & $\begin{array}{l}\text { K. pneumoniae } \\
(n=29)\end{array}$ & $\begin{array}{l}\text { P. aeruginosa } \\
(\mathrm{n}=23)\end{array}$ & $\begin{array}{l}\text { S. aureus } \\
(n=16)\end{array}$ & $\begin{array}{l}\text { E. coli } \\
(n=8)\end{array}$ & $\begin{array}{l}\text { H. influenzae } \\
(\mathrm{n}=5)\end{array}$ \\
\hline & $\begin{array}{l}\mathbf{n} \\
\%\end{array}$ & $\begin{array}{l}\mathbf{n} \\
\%\end{array}$ & $\begin{array}{l}\mathbf{n} \\
\%\end{array}$ & $\begin{array}{l}\mathbf{n} \\
\%\end{array}$ & $\begin{array}{l}\mathbf{n} \\
\%\end{array}$ & $\begin{array}{l}\mathbf{n} \\
\%\end{array}$ \\
\hline OXA & $\begin{array}{l}19 \\
82.60\end{array}$ & - & - & $\begin{array}{l}13^{\mathrm{a}, \mathrm{b}} \\
81.25\end{array}$ & - & - \\
\hline AMC & $\begin{array}{l}5^{c} \\
21.74\end{array}$ & $\begin{array}{l}28 \\
96.55\end{array}$ & - & - & $\begin{array}{l}6 \\
75\end{array}$ & $\begin{array}{l}0 \\
0\end{array}$ \\
\hline AMP/SAM & \begin{tabular}{|l}
5 \\
21.74 \\
\end{tabular} & $\begin{array}{l}27 \\
93.10 \\
\end{array}$ & - & - & $\begin{array}{l}6 \\
75 \\
\end{array}$ & $\begin{array}{l}0 \\
0 \\
\end{array}$ \\
\hline TZP & $\begin{array}{l}5^{c} \\
21.74\end{array}$ & $\begin{array}{l}1 \\
3.45\end{array}$ & $\begin{array}{l}11 \\
47.83\end{array}$ & - & $\begin{array}{l}4 \\
50\end{array}$ & - \\
\hline CXM & $\begin{array}{l}9 \\
39.13\end{array}$ & $\begin{array}{l}26 \\
87.7\end{array}$ & - & - & $\begin{array}{l}6 \\
75\end{array}$ & $\begin{array}{l}1 \\
20\end{array}$ \\
\hline CTX & $\begin{array}{l}5 \\
21.73\end{array}$ & $\begin{array}{l}19 \\
65.52\end{array}$ & - & - & $\begin{array}{l}5 \\
62.5\end{array}$ & $\begin{array}{l}0 \\
0\end{array}$ \\
\hline CRO & $\begin{array}{l}5 \\
21.73\end{array}$ & $\begin{array}{l}11 \\
37.93\end{array}$ & - & - & $\begin{array}{l}5 \\
62.5\end{array}$ & $\begin{array}{l}0 \\
0\end{array}$ \\
\hline CAZ & - & $\begin{array}{l}12 \\
41.38\end{array}$ & $\begin{array}{l}7 \\
30.43\end{array}$ & - & $\begin{array}{l}3 \\
37.5\end{array}$ & $\begin{array}{l}0 \\
0\end{array}$ \\
\hline FEP & $\begin{array}{l}3 \\
13.04\end{array}$ & $\begin{array}{l}11 \\
37.93\end{array}$ & $\begin{array}{l}8 \\
34.78\end{array}$ & - & $\begin{array}{l}4 \\
50\end{array}$ & $\begin{array}{l}0 \\
0\end{array}$ \\
\hline ETP & $\begin{array}{l} \\
4.35\end{array}$ & $\begin{array}{l} \\
3.45\end{array}$ & - & - & $\begin{array}{l}0 \\
0\end{array}$ & $\begin{array}{l}0 \\
0\end{array}$ \\
\hline MEM & $\begin{array}{l}2 \\
8.70\end{array}$ & $\begin{array}{l}2 \\
6.90\end{array}$ & $\begin{array}{l}6 \\
26.09\end{array}$ & - & $\begin{array}{l}0 \\
0\end{array}$ & $\begin{array}{l}0 \\
0\end{array}$ \\
\hline IPM & $\begin{array}{l}1 \\
4.35\end{array}$ & $\begin{array}{l}6 \\
20.70\end{array}$ & $\begin{array}{l}1 \\
4.35\end{array}$ & - & 0 & 0 \\
\hline VAN $^{d}$ & $\begin{array}{l}7 \\
30.43\end{array}$ & - & - & $\begin{array}{l}0 \\
0\end{array}$ & - & - \\
\hline AMK & - & $\begin{array}{l}8 \\
27.59\end{array}$ & $\begin{array}{l}9 \\
39.13 \\
\end{array}$ & $\begin{array}{l}8 \\
50 \\
\end{array}$ & $\begin{array}{l}1 \\
12.5 \\
\end{array}$ & - \\
\hline CIP & $\begin{array}{l}15 \\
65.22\end{array}$ & $\begin{array}{l}12 \\
41.38\end{array}$ & $\begin{array}{l}11 \\
47.83\end{array}$ & $\begin{array}{l}10 \\
62.5\end{array}$ & $\begin{array}{l}2 \\
25\end{array}$ & $\begin{array}{l}0 \\
0\end{array}$ \\
\hline LVX & $\begin{array}{l}3 \\
13.04\end{array}$ & $\begin{array}{l}10 \\
34.48\end{array}$ & $\begin{array}{l}13 \\
56.52\end{array}$ & $\begin{array}{l}5 \\
31.25\end{array}$ & $\begin{array}{l}3 \\
37.5\end{array}$ & 0 \\
\hline MOX & $\begin{array}{l}0 \\
0\end{array}$ & - & - & $\begin{array}{l}1 \\
6.25\end{array}$ & - & 0 \\
\hline AZM & $\begin{array}{l}11 \\
47.83\end{array}$ & - & - & $\begin{array}{l}9 \\
56.25\end{array}$ & - & $\begin{array}{l}1 \\
20\end{array}$ \\
\hline CLI & $\begin{array}{l}20 \\
86.96\end{array}$ & - & - & $\begin{array}{l}11 \\
68.75\end{array}$ & - & - \\
\hline SXT & $\begin{array}{l}23 \\
100\end{array}$ & $\begin{array}{l}26 \\
89.66\end{array}$ & - & $\begin{array}{l}8 \\
50\end{array}$ & $\begin{array}{l}5 \\
62.5\end{array}$ & \begin{tabular}{|l|}
2 \\
40
\end{tabular} \\
\hline
\end{tabular}

Notes: a Cefoxitin is tested as a surrogate for oxacillin. ${ }^{b} \mathrm{O}$ xacillin (cefoxitin)-resistant staphylococci are resistant to all tested $\beta$-lactam antimicrobial agents, Oxacillin (cefoxitin)-susceptible staphylococci can be considered susceptible to $\beta$-lactam/ $\beta$-lactamase inhibitor combinations, oral and parenteral cephems including cephalosporins I, II, III, and IV and carbapenems. 'Susceptibility inferred from the MIC of ampicillin. ${ }^{\circledR} S$. aureus isolates were tested for vancomycin susceptibility by E-test.

Abbreviations: AMC, amoxicillin/clavulanate; AMK, amikacin; AMP/SAM, ampicillin + sulbactam; AZM, azithromycin; CAZ, ceftazidime; CLI, clindamycin; CIP, ciprofloxacin; CRO, ceftriaxone; CTX, cefotaxime; CXM, cefuroxime; ETP, ertapenem; FEP, cefepime; IMP, imipenem; LVX, levofloxacin; MEM, meropnem; MOX, moxifloxacin; OXA, oxacillin; SXT, trimethoprim -sulfamethoxazole; TZP, piperacillin/tazobactam; VAN, vancomycin; S. Pneumoniae; Streptococcus pneumoniae; K. pneumoniae, Klebsiella pneumoniae; P. aeruginosa, Pseudomonas aeruginosa; S. aureus, Staphylococcus aureus; E. coli, Escherichia coli; H. influenzae, Haemophilus influenzae.

to ciprofloxacin and levofloxacin, respectively. Resistance rates in K. pneumoniae for quinolones and $\beta$-lactams were higher than those reported for $K$. pneumoniae CAP isolates in Egypt. ${ }^{17}$ Lin et $\mathrm{al}^{20}$ and Sikarwar and Barta ${ }^{21}$ published analogous data.
High rates of antibiotic resistance were detected among S. pneumoniae, where $82.6 \%$ of $S$. pneumoniae isolates were recorded as resistant to oxacillin; all showed penicillin MIC values $>0.12 \mu \mathrm{g} / \mathrm{mL}$, and $47.83 \%$ were resistant to azithromycin. This is in accordance with the Egyptian as 
Table 4 Comparison of responders' and nonresponders' characteristics

\begin{tabular}{|c|c|c|c|c|c|}
\hline \multirow[t]{2}{*}{ Characteristics } & \multicolumn{2}{|c|}{$\begin{array}{l}\text { Nonresponders } \\
(n=43)\end{array}$} & \multicolumn{2}{|c|}{$\begin{array}{l}\text { Responders } \\
(n=227)\end{array}$} & \multirow[t]{2}{*}{$P$-value } \\
\hline & $\mathbf{n}$ & $\%$ & $\mathbf{n}$ & $\%$ & \\
\hline Age in years (mean $\pm S D)$ & \multicolumn{2}{|c|}{$53.4 \pm 13$} & \multicolumn{2}{|c|}{$58.8 \pm 14.2$} & $0.02 *$ \\
\hline Gender & & & & & \\
\hline Male $(n=180)$ & 28 & 15.56 & 152 & 84.44 & 0.81 \\
\hline Female $(n=90)$ & 15 & 16.67 & 75 & 83.33 & \\
\hline Smokers $(n=142)$ & 23 & 16.20 & 119 & 83.8 & 0.89 \\
\hline Comorbidities $(n=108)$ & 21 & 19.44 & 87 & 80.56 & 0.2 \\
\hline Diabetes mellitus ( $n=34$ ) & 15 & 44.11 & 19 & 55.89 & $<0.001 *$ \\
\hline Hypertension ( $n=28$ ) & 9 & 32.14 & 19 & 67.86 & $0.01 *$ \\
\hline Ischemic heart disease $(n=18)$ & 7 & 38.88 & 11 & 61.12 & $0.006^{*}$ \\
\hline Liver disease $(n=8)$ & 4 & 50 & 4 & 50 & $0.008^{*}$ \\
\hline $\operatorname{COPD}(n=20)$ & 7 & 35 & 13 & 65 & $0.015^{*}$ \\
\hline \multicolumn{6}{|l|}{ Initial empiric antimicrobial treatment } \\
\hline -Levofloxacin $(n=33)$ & 7 & 21.21 & 26 & 78.79 & 0.38 \\
\hline -Antipneumococcal $\beta$-lactam+ azithromycin $(n=87)$ & 4 & 4.60 & 83 & 95.40 & $<0.001 *$ \\
\hline -Antipneumococcal $\beta$-lactam+ levofloxacin $(n=67)$ & $\mathrm{II}$ & 16.41 & 50 & 83.59 & 0.61 \\
\hline -Anti-MRSA coverage $(n=3 \mid)^{a}$ & 5 & 16.12 & 26 & 83.87 & 0.97 \\
\hline \multicolumn{6}{|l|}{-Anti-pseudomonal coverage } \\
\hline Anti-pseudomonal/anti-pneumoccocal $\beta$-lactam + amikacin + azithromycin $(n=29)$ & 3 & 10.34 & 26 & 89.66 & 0.38 \\
\hline Anti-pseudomonal/anti-pneumoccocal $\beta$-lactam + ciprofloxacin/levofloxacin $(n=23)$ & 7 & 30.43 & 16 & 69.57 & 0.05 \\
\hline \multicolumn{6}{|l|}{ Bacterial agent identified/case ${ }^{b}$} \\
\hline Cases with no bacteria identified $(n=134)$ & 6 & 4.48 & 128 & 95.52 & $<0.001 *$ \\
\hline \multicolumn{6}{|l|}{ Cases with single bacterial agent $(n=127)$} \\
\hline S. pneumoniae $(n=2 \mathrm{I})$ & 4 & 19.05 & 17 & 80.95 & 0.7 \\
\hline K. pneumoniae $(\mathrm{n}=28)$ & 10 & 35.71 & 18 & 64.29 & $0.003^{*}$ \\
\hline P. aeruginosa $(\mathrm{n}=2 \mathrm{I})$ & 9 & 42.86 & 12 & 57.14 & $<0.001 *$ \\
\hline S.aureus $(\mathrm{n}=\mathrm{II})$ & 5 & 45.45 & 6 & 54.55 & $0.006^{*}$ \\
\hline Mixed bacteria $(n=9)$ & 5 & 55.56 & 4 & 44.44 & $<0.00 I^{*}$ \\
\hline M. tuberculosis $(n=4)$ & 4 & 100 & 0 & 0 & $<0.001 *$ \\
\hline \multicolumn{6}{|l|}{ CURB-65 severity score } \\
\hline Class $2(n=185)$ & 25 & $|3.5|$ & 160 & 86.49 & 0.11 \\
\hline Classes $3-5(n=85)$ & 18 & 21.18 & 67 & 78.82 & \\
\hline \multicolumn{6}{|l|}{ Complications } \\
\hline Suppurative complications ${ }^{d}(n=47)$ & 9 & 19.15 & 38 & 80.85 & 0.77 \\
\hline Shock $(n=25)$ & 4 & 16 & 21 & 84 & \\
\hline Respiratory failure $(n=25)$ & 6 & 24 & 19 & 76 & \\
\hline
\end{tabular}

Notes: ${ }^{2}$ Vancomycin was added. ${ }^{~}$ The following bacteria are not presented in the table because all of them were isolated from responding cases: $E$. coli (8 cases), $H$. influenzae ( 5 cases), M. pneumoniae (15 cases), L. pneumophilia (10 cases), C. pneumoniae (4 cases). 'Empyema or abscess, $* P<0.05$ indicates significant relation. Values are mean \pm SD, or $n(\%)$. Abbreviations: CURB-65 severity score [C, mental confusion; U, blood urea $>7 \mathrm{mmol} / \mathrm{L}$; R, respiratory rate $\geq 30 / \mathrm{min}$; B, low blood pressure (diastolic $\leq 60 \mathrm{mmHg}$ or systolic $<90 \mathrm{mmHg}$ ); age $\geq 65$ years] MRSA, amethicillin resistant Staphylococcus aureus; S. Pneumoniae; Streptococcus pneumoniae; K. pneumoniae, Klebsiella pneumoniae; $P$. aeruginosa, Pseudomonas aeruginosa; S. aureus, Staphylococcus aureus; E. coli, Escherichia coli; H. influenzae, Haemophilus influenzae; M. pneumoniae, Mycoplasma pneumoniae; L. pneumophila, Legionella pneumophila; C. pneumoniae, Chlamydophila pneumoniae; M. tuberculosis, Mycobacterium tuberculosis.

well as the global trend of increasing resistance of $S$. pneumoniae to $\beta$-lactams and macrolides; Bahy et $\mathrm{al}^{22}$ in a recent Egyptian study reported high rates of resistance to penicillin (80\% and $82 \%)$ and macrolides (73\% and $78 \%$ ) in the two predominant $S$. pneumoniae serotypes $6 \mathrm{~A} / \mathrm{B}$ and $19 \mathrm{~F}$ which exceeded those reported from older Egyptian studies. ${ }^{17,23} \mathrm{~A}$ nearly similar pattern was reported by Mohammed et al. ${ }^{24} \mathrm{As}$ for quinolones, $65.2 \%$ and $13.04 \%$ of $S$. pneumoniae isolates were resistant to ciprofloxacin and levofloxacin, respectively, while all $(100 \%)$ retained susceptibility to moxifloxacin. This is explained by the fact that the older second generation fluoroquinolones (ciprofloxacin and levofloxacin) target only the ParC subunit of the enzyme topoisomerase IV, and prolonged exposure to these agents selected for resistant strains with mutated ParC region. Moxifloxacin, however, retains activity on such mutant strains by the virtue of its additional effect on the unaltered GyrA subunit of DNA gyrase. In clinical practice, the ideal pharmacodynamics and adequate tissue penetration are additional advantages of moxifloxacin over levofloxacin in pneumococcal CAP. ${ }^{25,26}$ 
P. aeruginosa showed considerable resistance rates to antibiotics with anti-pseudomonal activity. They showed the least susceptibility to ciprofloxacin, levofloxacin and piperacillin/tazobactam. Carbapenems (imipenem and meropenem) showed the highest susceptibility rates $(95.65 \%$ and $73.91 \%$, respectively), while resistance to amikacin was detected in $39.13 \%$. This is consistent with large-scale surveillance results $^{27,28}$ and Egyptian studies ${ }^{23,29}$ except for the higher rates of quinolones and amikacin resistance in the latter.

Treatment of $P$. aeruginosa infections is to some extent difficult owing to its low outer membrane permeability. Moreover, it has the capability of acquiring resistance to most antibiotics. A variety of mechanisms may be implicated; eg, production of efflux pumps, use of selective porins, and possessing inducible beta-lactamases. So, P. aeruginosa therapy is better guided by the results of the susceptibility reports of individual strains..$^{30}$

Our results pointed out that $81.25 \%$ of isolated S. aureus were MRSA. Previous Egyptian studies showed that MRSA represented $71 \%{ }^{22}$ and $79.3 \%{ }^{17}$ of S. aureus isolates. Vancomycin and moxifloxacin showed the highest susceptibility rates.

The MDR phenotype was a common finding among our isolates, where $76.2 \%$ of tested bacteria were MDR. In 2015, Mohamed et $\mathrm{al}^{24}$ reported MDR of $73.3 \%$ among S. pneumoniae in Egypt. Researchers from other countries reported comparable results; Li et a ${ }^{31}$ reported that $40 \%$ of $P$. aeruginosa, $62 \%$ of $E$. coli and $36 \%$ of $K$. pneumoniae were MDR. Cillóniz et $\mathrm{a}^{32}$ found that $32 \%$ of $P$. aeruginosa isolates were MDR. These results highlight the antibiotic resistance in the community as a problem which calls for strenuous efforts to rationalize antibiotic use and eliminate over-the-counter antibiotic dispensing and self-medication especially in developing countries. Moreover, it emphasizes a lack of antimicrobial stewardship and defective infection control practices.

Adequate antibiotic therapy usually results in some improvement in the patient's clinical course within 48-72 hours. If no improvement was observed, patients are considered nonresponders and are at high risk of in-hospital death. ${ }^{33}$ Forty-three (15.9\%) CAP patients in the current study were nonresponders. Many causes may lead to nonresponsiveness. Patient-related factors (including suppurative complications), medication-related factors and resistant bacteria are important causes. ${ }^{34}$

While investigating the response to each used empiric antibiotic regimen, the highest rate of nonresponsiveness was observed in cases receiving anti-pseudomonal/antipneumoccocal $\beta$-lactam plus ciprofloxacin/levofloxacin
$30.43 \%$ (seven out of 23 cases received this regimen for anti-pseudomonal coverage) followed by those receiving an antipneumococcal $\beta$-lactam plus levofloxacin 11/67 (16.41\%) and anti-MRSA 5/31 (16.12\%) for severe CAP, but did not reach statistical significance. Meanwhile azithromycin containing regimens showed the lowest rates of nonresponsiveness (4.60\%) and (10.34\%) for antipneumococcal $\beta$-lactam plus azithromycin combination and antipseudomonal /anti-pneumoccocal $\beta$-lactam plus amikacin plus azithromycin combination, respectively. Several studies reported a benefit inferred by adding a macrolide to empiric combinations in treatment of CAP, even those caused by macrolide insensitive isolates, particularly in ICU admitted patients with sepsis. ${ }^{35-37}$ This benefit stems from the anti-inflammatory properties of macrolides rather than the antimicrobial ones. Macrolides can decrease the chemotactic response of neutrophils, promote macrophage phagocytosis of apoptotic cells, enhance the release of anti-inflammatory cytokines, inhibit the synthesis of proinflammatory cytokines and reduce T-cell numbers and migration. ${ }^{38,39}$ Although macrolide and fluoroquinolones containing combinations have been generally comparable in different clinical trials, many observational studies have reported better clinical outcomes with macrolide containing regimens for patients with severe CAP. Thus, these results should be interpreted with caution and need to be proven by well-designed clinical trials considering specific patient and disease characteristics.

Bacterial etiology was identified in 37 nonresponders (86\%). S. pneumoniae, K pneumoniae, P. aeruginosa, $S$. aureus, mixed bacteria and Mycobacteria were the bacterial agents identified at rates comparable to Bodi et al. ${ }^{40}$ Similarly, other researchers observed that nonresponding pneumonia was mostly due to organisms not covered by initial empirical antibiotic therapy; this applies to MDR organisms, atypical bacteria as well as mycobacterial tuberculosis. ${ }^{18,39,41-44}$ Mixed bacterial etiology was another contributing factor to nonresponsiveness to initial empiric therapy, where a statistically significant difference was recorded, by the research team, between responders and nonresponders for the presence of mixed infection. Nonresponders in the current study showed a higher mortality rate (16.3\%) compared to responders $(4.85 \%)$. These findings were similar to a previous study, in which early nonresponse was identified in $8.4 \%$ of CAP patients, with mortality rate of $24 \% .{ }^{45}$ Another CAP cohort showed a nonresponder rate of $15.9 \%$ and an in-hospital mortality of $17.3 \%$ in nonresponders compared to $5.2 \%$ in responders. ${ }^{46}$ 


\section{Conclusion}

Local resistance statistics is very important to avoid the risk of inadequate therapy. Bacterial profile should be updated regularly as some nosocomial pathogens have emerged in the community causing pneumonia. The growing prevalence of MDR bacteria represents an important issue in choosing empiric antimicrobial management in seriously ill hospitalized patients. The widespread antibiotic-resistant microorganisms necessitate the implementation of antibiotic stewardship strategies, including de-escalation, shifting to oral therapy, rapid patient ambulation and discharge, and shorter duration of antibiotic therapy, which rely on evaluation of patient responses to initial empiric therapy. Factors related to bacterial agents, the antibiotic treatment, the host and their interactions may lead to failed treatment protocol. P. aeruginosa, tuberculosis and mixed agents should be considered in nonresponders. Broad spectrum $\beta$-lactams, especially carbapenems, and moxifloxacin showed in vitro efficacy on most of the tested isolates. Advanced macrolides (azithromycin) containing regimens showed the lowest rates of nonresponsiveness, have the advantage of atypical coverage and can spare fluoroquinolones as important second-line anti-tuberculous agents in patients at risk of TB especially MDR-TB. However, this result should be interpreted with caution and supported by further studies.

\section{Recommendations}

The antibiotic stewardship program is a necessity; further studies are needed to monitor its implementation. Future studies are needed to explore the molecular basis of the reported resistance patterns. The response to initial empiric treatment could be further investigated on a larger scale with each of the recorded associated comorbid conditions.

\section{Limitations of the study}

Antibiotic susceptibility testing for atypical bacteria was not feasible. Viral causes were not explored by this research, although the clinicians requested test for viral causes of CAP as a part of their diagnostic approach of the disease, which could be the objective of future studies with different scopes. The lack of antibiotic stewardship programs in the investigated hospital hinders proper stratification of patients. The shortage of national surveillance data limits the detailed interpretation of results.

\section{Acknowledgments}

The authors appreciate the nursing staff at the chest department for their cooperation during specimen collection.

\section{Disclosure}

The authors report no conflicts of interest in this work.

\section{References}

1. Jain S, Self WH, Wunderink RG, et al. Community-Acquired Pneumonia Requiring Hospitalization among U.S. Adults. $N$ Engl J Med. 2015;373(5):415-427.

2. Howard LS, Sillis M, Pasteur MC, Kamath AV, Harrison BD. Microbiological profile of community-acquired pneumonia in adults over the last 20 years. $J$ Infect. 2005;50(2):107-113.

3. Musher DM, Thorner AR. Community-acquired pneumonia. $N$ Engl J Med. 2014;371(17):1619-1628.

4. International Organization for Standards (ISO 20776-1). Clinical laboratory testing and in vitro diagnostic test systems. Susceptibility Testing of Infectious Agents and Evaluation of Performance of Antimicrobial Susceptibility Testing Devices. Part 1. Reference method for testing the in vitro activity of antimicrobial agents against rapidly growing aerobic bacteria involved in infectious diseases. Geneva, Switzerland: International Organization for Standards; 2006.

5. Mandell LA, Wunderink RG, Anzueto A, et al. Infectious Diseases Society of America/American Thoracic Society consensus guidelines on the management of community-acquired pneumonia in adults. Clin Infect Dis. 2007;44(Suppl 2):S27-S72.

6. National Clinical Guideline Centre (UK) [database on the Internet] Pneumonia: Diagnosis and Management of Community- and HospitalAcquired Pneumonia in Adults. London: National Institute for Health and Care Excellence (UK); 2014 Dec. (NICE Clinical Guidelines, No. 191.). Available from: https://www.ncbi.nlm.nih.gov/books/ NBK263426/. Accessed October 3, 2018.

7. Liapikou A, Ferrer M, Polverino E, et al. Severe community-acquired pneumonia: validation of the Infectious Diseases Society of America/ American Thoracic Society guidelines to predict an intensive care unit admission. Clin Infect Dis. 2009;48(4):377-385.

8. Lim WS, van der Eerden MM, Laing R, et al. Defining community acquired pneumonia severity on presentation to hospital: an international derivation and validation study. Thorax. 2003;58(5):377-382.

9. Cheesbrough M. Microbiological tests. District Laboratory Practice in Tropical Countries. Cambridge: Cambridge University Press; 2005:62-70.

10. CLSI. Performance Standards for Antimicrobial Susceptibility Testing, Twenty-Fifth Informational Supplement. CLSI document M100-S25. Wayne, PA: Clinical and Laboratory Standards Institute; 2015.

11. Magiorakos AP, Srinivasan A, Carey RB, et al. Multidrug-resistant, extensively drug-resistant and pandrug-resistant bacteria: an international expert proposal for interim standard definitions for acquired resistance. Clin Microbiol Infect. 2012;18(3):268-281.

12. Richter SS, Heilmann KP, Dohrn CL, Riahi F, Beekmann SE, Doern GV. Changing epidemiology of antimicrobial-resistant Streptococcus pneumoniae in the United States, 2004-2005. Clin Infect Dis. 2009;48(3):e23-e33.

13. Lodise TP, Anzueto AR, Weber DJ, et al. Assessment of time to clinical response, a proxy for discharge readiness, among hospitalized patients with community-acquired pneumonia who received either ceftaroline fosamil or ceftriaxone in two phase III FOCUS trials. Antimicrob Agents Chemother. 2015;59(2):1119-1126.

14. Millett ER, De Stavola BL, Quint JK, Smeeth L, Thomas SL. Risk factors for hospital admission in the 28 days following a communityacquired pneumonia diagnosis in older adults, and their contribution to increasing hospitalisation rates over time: a cohort study. BMJ Open. 2015;5(12):e008737.

15. Confalonieri M, Urbino R, Potena A, et al. Hydrocortisone infusion for severe community-acquired pneumonia: a preliminary randomized study. Am J Respir Crit Care Med. 2005;171(3):242-248.

16. Khalil MM, Abdel Dayem AM, Farghaly AAA-H, Shehata HM. Pattern of community and hospital acquired pneumonia in Egyptian military hospitals. Egypt J Chest Dis Tuberc. 2013;62(1):9-16. 
17. Agmy G, Mohamed S, Gad Y, Farghally E, Mohammedin H, Rashed H. Bacterial profile, antibiotic sensitivity and resistance of lower respiratory tract infections in upper egypt. Mediterr J Hematol Infect Dis. 2013;5(1):e2013056.

18. Rodrigo-Troyano A, Sibila $\mathrm{O}$. The respiratory threat posed by multidrug resistant Gram-negative bacteria. Respirology. 2017;22(7):1288-1299.

19. Grosso A, Famiglietti A, Luna CM. Community-acquired pneumonia due to gram-negative bacteria. Community Acquir Infect. 2015;2(4):117-122.

20. Lin WP, Wang JT, Chang SC, et al. The Antimicrobial Susceptibility of Klebsiella pneumoniae from Community Settings in Taiwan, a Trend Analysis. Sci Rep. 2016;6(1):1-11.

21. Sikarwar AS, Batra HV. Prevalence of antimicrobial drug resistance of Klebsiella pneumoniae in India. Int J Biosci Biochem Bioinforma. 2011;1(3):211-215.

22. Bahy RH, Hamouda HM, Shahat AS, Yassin AS, Amin MA. Serotype identification and antibiotic resistance of the predominant Streptococcus pneumoniae in Egypt. Der Pharm Lett. 2015;7(11):166-171.

23. El Kholy A, Baseem H, Hall GS, Procop GW, Longworth DL. Antimicrobial resistance in Cairo, Egypt 1999-2000: a survey of five hospitals. J Antimicrob Chemother. 2003;51(3):625-630.

24. Mohammed NM, Badr MF, El Nagdy MM, Soliman OE, El Nady GM. Macrolide resistant genotypes of pneumococcal isolates in Mansoura University Children's Hospital. Egypt J Med Microbiol. 2015;24(1):7-14.

25. Patel SN, McGeer A, Melano R, et al. Susceptibility of Streptococcus pneumoniae to fluoroquinolones in Canada. 2011;55(8):3703-3708.

26. Bolon MK. The newer fluoroquinolones. Infect Dis Clin North Am. 2009;23(4):1027-1051.

27. Flamm RK, Weaver MK, Thornsberry C, Jones ME, Karlowsky JA, Sahm DF. Factors associated with relative rates of antibiotic resistance in Pseudomonas aeruginosa isolates tested in clinical laboratories in the United States from 1999 to 2002. Antimicrob Agents Chemother. 2004:48(7):2431-2436.

28. Obritsch MD, Fish DN, MacLaren R, Jung R. National surveillance of antimicrobial resistance in Pseudomonas aeruginosa isolates obtained from intensive care unit patients from 1993 to 2002. Antimicrob Agents Chemother. 2004;48(12):4606-4610.

29. Labah EA, Afifi IK, Ahmed LMS. Community-Acquired Urinary Tract Infections in Tanta. Egypt: Aetiology and Antibiotic Resistance Pattern; 2009;18(4):179-190.

30. Juayang A, Lim J, Bonifacio A, et al. Five-Year Antimicrobial Susceptibility of Pseudomonas aeruginosa from a Local Tertiary Hospital in Bacolod City, Philippines. Trop Med Infect Dis. 2017;2(3):28.

31. Li XJ, Li Q, Si LY, Yuan QY. Bacteriological differences between COPD exacerbation and community-acquired pneumonia. Respir Care. 2011;56(11):1818-1824.

32. Cillóniz C, Gabarrús A, Ferrer M, et al. Community-Acquired Pneumonia Due to Multidrug- and Non-Multidrug-Resistant Pseudomonas aeruginosa. Chest. 2016;150(2):415-425.
33. Gonçalves-Pereira J, Conceição C, Póvoa P. Community-acquired pneumonia: identification and evaluation of nonresponders. Ther $A d v$ Infect Dis. 2013;1(1):5-17.

34. Genné D, Sommer R, Kaiser L, et al. Analysis of factors that contribute to treatment failure in patients with community-acquired pneumonia. Eur J Clin Microbiol Infect Dis. 2006;25(3):159-166.

35. Cilloniz C, Albert RK, Liapikou A, et al. The Effect of Macrolide Resistance on the Presentation and Outcome of Patients Hospitalized for Streptococcus pneumoniae Pneumonia. Am J Respir Crit Care Med. 2015;191(11):1265-1272.

36. Zhanel GG, Wolter KD, Calciu C, et al. Clinical cure rates in subjects treated with azithromycin for community-acquired respiratory tract infections caused by azithromycin-susceptible or azithromycin-resistant Streptococcus pneumoniae: analysis of Phase 3 clinical trial data. J Antimicrob Chemother. 2014;69(10):2835-2840.

37. Sligl WI, Asadi L, Eurich DT, Tjosvold L, Marrie TJ, Majumdar SR. Macrolides and mortality in critically ill patients with communityacquired pneumonia: a systematic review and meta-analysis. Crit Care Med. 2014;42(2):420-432.

38. Zarogoulidis P, Papanas N, Kioumis I, Chatzaki E, Maltezos E, Zarogoulidis K. Macrolides: from in vitro anti-inflammatory and immunomodulatory properties to clinical practice in respiratory diseases. Eur J Clin Pharmacol. 2012;68(5):479-503.

39. Altenburg J, de Graaff CS, van der Werf TS, Boersma WG. Immunomodulatory effects of macrolide antibiotics - part 1: biological mechanisms. Respiration. 2011;81(1):67-74.

40. Bodí M, Rodríguez A, Solé-Violán J, et al. Antibiotic prescription for community-acquired pneumonia in the intensive care unit: impact of adherence to Infectious Diseases Society of America guidelines on survival. Clin Infect Dis. 2005;41(12):1709-1716.

41. Finch S, Chalmers JD. Brief Clinical Review: Non-Responding Pneumonia. Eur Med J. 2014;2(October):104-111.

42. Micek ST, Dunne M, Kollef MH. Pleuropulmonary complications of Panton-Valentine leukocidin-positive community-acquired methicillin-resistant Staphylococcus aureus: importance of treatment with antimicrobials inhibiting exotoxin production. Chest. 2005;128(4): $2732-2738$

43. Cillóniz C, Ewig S, Ferrer M, et al. Community-acquired polymicrobial pneumonia in the intensive care unit: aetiology and prognosis. Crit Care. 2011;15(5):R209.

44. Arancibia F, Ewig S, Martinez JA, et al. Antimicrobial treatment failures in patients with community-acquired pneumonia: causes and prognostic implications. Am J Respir Crit Care Med. 2000;162(1):154-160.

45. Menéndez R, Cavalcanti M, Reyes S, et al. Markers of treatment failure in hospitalised community acquired pneumonia. Thorax. 2008;63(5): $447-452$.

46. Ott SR, Hauptmeier BM, Ernen C, et al. Treatment failure in pneumonia: impact of antibiotic treatment and cost analysis. Eur Respir J. 2012;39(3):611-618
Infection and Drug Resistance

\section{Publish your work in this journal}

Infection and Drug Resistance is an international, peer-reviewed openaccess journal that focuses on the optimal treatment of infection (bacterial, fungal and viral) and the development and institution of preventive strategies to minimize the development and spread of resistance. The journal is specifically concerned with the epidemiology of antibiotic

\section{Dovepress}

resistance and the mechanisms of resistance development and diffusion in both hospitals and the community. The manuscript management system is completely online and includes a very quick and fair peerreview system, which is all easy to use. Visit http://www.dovepress.com/ testimonials.php to read real quotes from published authors. 